\title{
Estudo do metabolismo de rotina e excreção de amônia do anfípoda antártico Waldeckia obesa em duas temperaturas distintas
}

\author{
Vicente GOMES; PHAN Van Ngan \& Maria José de Arruda Campos Rocha PASSOS \\ Instituto Oceanográfico da Universidade de São Paulo \\ (Caixa Postal 66149, 05389-970, São Paulo, SP, Brasil)
}

- Abstract: Mean and specific oxygen consumption and ammonia excretion were measured at $0^{\circ} \mathrm{C}$ and $3^{\circ} \mathrm{C}$, in the Antarctic amphipod Waldeckia obesa collected in Admiralty Bay, King George Island, Antarctica. Experiments were carried out at the Brazilian Antarctic Station "Comandante Ferraz" and at the Laboratory of Polar Ecology of the Oceanographic Institute of São Paulo University. Individuals ranging from $120 \mathrm{mg}$ to $620 \mathrm{mg}$ and from 40 $\mathrm{mg}$ to $690 \mathrm{mg}$ were employed in experiments at $0^{\circ} \mathrm{C}$ and $3^{\circ} \mathrm{C}$, respectively. Within this range of weight, mean oxygen consumption varied from $2.22 \mu \mathrm{l} / \mathrm{h}$ to $10.81 \mu \mathrm{l} / \mathrm{h}$ and specific consumption from $0.011 \mu \mathrm{l} / \mathrm{mg} / \mathrm{h}$ to $0.018 \mu \mathrm{l} / \mathrm{mg} / \mathrm{h}$, at $0^{\circ} \mathrm{C}$. At $3^{\circ} \mathrm{C}$, mean oxygen consumption varied from $1.83 \mu \mathrm{l} / \mathrm{h}$ to $14.9 \mu \mathrm{l} / \mathrm{h}$ and specific consumption from $0.033 \mu \mathrm{l} / \mathrm{mg} / \mathrm{h}$ to 0.022 $\mu \mathrm{l} / \mathrm{mg} / \mathrm{h}$. Q10 value calculated from average oxygen consumption was 6.95 . Mean ammonia excretion, at $0^{\circ} \mathrm{C}$, varied from $13.84 \mathrm{ng}$.at $/ \mathrm{h}$ to $55.34 \mathrm{ng}$.at $/ \mathrm{h}$ and specific excretion from 0.090 ng.at $/ \mathrm{mg} / \mathrm{h}$ to $0.042 \mathrm{ng} \cdot \mathrm{at} / \mathrm{mg} / \mathrm{h}$. At $3^{\circ} \mathrm{C}$, mean ammonia excretion varied from $5.11 \mathrm{ng}$.at $/ \mathrm{h}$ to $38.33 \mathrm{ng} . \mathrm{at} / \mathrm{h}$ and specific excretion from $0.088 \mathrm{ng} . \mathrm{at} / \mathrm{mg} / \mathrm{h}$ to $0.059 \mathrm{ng} \cdot \mathrm{at} / \mathrm{mg} / \mathrm{h}$. O:N ratio indicates that a mixture of protein and lipids is utilized for catabolism. At $3^{\circ} \mathrm{C}$, however, there is a tendency to increase the lipid contribution for the substrate. Oxygen consumption of Waldeckia obesa at $0^{\circ} \mathrm{C}$ is very low and is in accordance with the values reported by some other authors. These data and others recent results found in the literature indicate the fragility of the "metabolic cold adaptation" hypothesis, that assumes that marine Antarctic ectotherms have high metabolic rates, as an evolutive adaptation to low temperatures. Oxygen consumption and ammonia excretion data could be extrapolated to populational parameters giving support for the studies on the ecological role of these animals in their ecosystem.

- Resumo: Realizaram-se estimativas, a $0^{\circ} \mathrm{C}$ e a $3^{\circ} \mathrm{C}$, do consumo médio e específico de oxigênio e da excreção média e específica de amônia de anfípodas antárticos da espécie Waldeckia obesa, coletados na Baía do Almirantado, Ilha Rei George, Antártica. Os experimentos foram realizados na Estação Antártica Brasileira "Comandante Ferraz" e no Laboratório de Ecologia Polar do Instituto Oceanográfico da Universidade de São Paulo. Foram empregados animais entre $120 \mathrm{mg}$ a $620 \mathrm{mg}$ e entre $40 \mathrm{mg}$ e $690 \mathrm{mg}$ de peso úmido, nos experimentos a $0^{\circ} \mathrm{C}$ e a $3^{\circ} \mathrm{C}$, respectivamente. Dentro dessas faixas de peso, o consumo médio de oxigênio, a $0^{\circ} \mathrm{C}$, variou de $2,22 \mu \mathrm{l} / \mathrm{h}$ a $10,81 \mu \mathrm{l} / \mathrm{h}$ e o específico de $0,011 \mu \mathrm{l} / \mathrm{mg} / \mathrm{h}$ a $0,018 \mu \mathrm{l} / \mathrm{mg} / \mathrm{h}$. A $3^{\circ} \mathrm{C}$, o consumo médio de oxigênio variou de $1,83 \mu \mathrm{l} / \mathrm{h}$ a $14,19 \mu \mathrm{l} / \mathrm{h}$ e o específico de $0,033 \mu \mathrm{l} / \mathrm{mg} / \mathrm{h} \mathrm{a} 0,022 \mu \mathrm{l} / \mathrm{mg} / \mathrm{h}$. O Q10, calculado a partir das médias das classes, foi de 6,95 . Por sua vez, a excreção média de amônia, a $0^{\circ} \mathrm{C}$, variou de $13,84 \mathrm{ng}$.at/h a 55,34 ng.at $/ \mathrm{h}$ e a específica entre $0,090 \mathrm{ng} . \mathrm{at} / \mathrm{mg} / \mathrm{h}$ a $0,042 \mathrm{ng} . \mathrm{at} / \mathrm{mg} / \mathrm{h}$. A $3^{\circ} \mathrm{C}$, a excreção média de amônia variou de $5,11 \mathrm{ng}$.at $/ \mathrm{h}$ a $38,33 \mathrm{ng}$.at/h e a específica de $0,088 \mathrm{ng} \cdot \mathrm{at} / \mathrm{mg} / \mathrm{h}$ a 0,059 ng.at $/ \mathrm{mg} / \mathrm{h}$. A relação $\mathrm{O}: \mathrm{N}$ indica que uma mistura de proteínas e lipídios é utilizada como substrato para o catabolismo. A $3^{\circ} \mathrm{C}$, entretanto, há uma tendência a aumentar a contribuiçẫo dos lipídios nesse substrato. O consumo de oxigênio de Waldeckia obesa é bastante baixo estando de acordo com os valores encontrados, a $0^{\circ} \mathrm{C}$, por outros autores. Esses dados, juntamente com resultados recentes encontrados na literatura, indicam a fragilidade da hipótese de "adaptação metabólica ao frio", que supōe terem os animais marinhos ectotérmicos antárticos taxas metabólicas elevadas, como resposta adaptativa às baixas temperaturas. Os dados de consumo de oxigênio e excreção de amônia fornecem material básico para extrapolação a parâmetros populacionais, fornecendo subsídios para a avaliaçāo do papel ecológico desses animais no ecossistema em que vivem.

- Descriptors: Amphipods, Metabolism, Antarctica, Waldeckia obesa, Oxygen consumption, Excretion.

- Descritores: Anfípodas, Metabolismo, Antártica, Waldeckia obesa, Consumo de oxigênio, Excreçāo. 


\section{Introdução}

Estudos de metabolismo de anfípodas polares têm despertado o interesse dos pesquisadores tanto do ponto de vista fisiológico quanto do ecológico.

Do ponto de vista fisiológico, esses estudos têm procurado, principalmente, compreender os processos de adaptação desses animais aos fatores ambientais, sobretudo à temperatura. Resultados de diversos trabalhos levaram os autores a concluir que animais polares apresentam metabọlismo mais elevado do que seria de se esperar pela extrapolação do metabolismo dos animais de regiōes temperadas às temperaturas em que vivem os de regiôes polares. Esse fenômeno foi chamado de "metabolic cold adaptation", ou seja, "adaptação metabólica ao frio", sendo o termo empregado pela primeira vez em peixes (Wohlschlag, 1964). Conclusōes semelhantes foram encontradas para anfípodas polares (Armitage, 1962; Klekowski et al., 1973; Rakusa-Suszczewski \& Klekowski, 1973; Opalinski, 1979). Esses resultados, entretanto, foram revistos por vários autores sendo alvo de muitas críticas. Muitos pesquisadores acreditam, atualmente, que os dados que permitiram essas conclusões são resultantes de falhas metodológicas que superestimam as taxas metabólicas, principalmente por não tomarem os cuidados necessários para evitar o estresse dos animais utilizados nos experimentos (Holeton, 1974; Clarke, 1991).

Do ponto de vista ecológico, os anfípodas são animais de grande importância na cadeia trófica do ecossistema antártico, tanto nas regiōes costeiras quanto no mar aberto. Ocupam uma grande variedade de nichos tendo papel de destaque na reciclagem da matéria orgânica. Servem diretamente como fonte de alimento para invertebrados e vertebrados, tais como para cefalópodos, peixes, aves, etc. (Opalinski \& Jazdzewski, 1978; Jazdzewski, 1993). Em extensas regiōes das áreas livres de gelo do Oceano Antártico, anfípodas hiperídeos parecem exercer papel ecológico semelhante ao do krill (Hureau, 1994). Na Baía do Almirantado, região onde foi desenvolvido este trabalho, há cerca de 100 espécies descritas de anfípodas ocupando os mais diversos habitats e níveis tróficos (De Broyer \& Klages, 1990).

Por outro lado, o estudo do balanço energético de um organismo é um dos caminhos mais eficazes para se compreender seu papel ecológico. Ele expressa a proporção de energia consumida que é alocada aos vários processos vitais e mostra como esta pode variar durante o ano ou o ciclo de vida.

Waldeckia obesa é uma espécie circum-antártica da família Lysianassidae, de hábitos necrófagos, ocorrendo desde o litoral até $660 \mathrm{~m}$ de profundidade. Geralmente caminham sobre o fundo marinho, congregando-se em grande número sobre restos de animais dos quais se alimentam (Chapelle \& Peck, 1995). Foram reconhecidos como tendo grande importância ecológica sendo membros preponderantes do grupo dos animais necrófagos (Arnaud et al., 1986; De Broyer \& Klages, 1990) desempenhando, certamente, função relevante na reciclagem da matéria orgânica. É uma espécie que resiste bem em cativeiro tendo sido mantida em câmaras frias em São Paulo e em Bruxelas por mais de 3 anos. Algumas tentativas para estudar o metabolismo de Waldeckia obesa forneceram resultados bastante diferentes (Rakusa-Suszczewski \& Lach, 1991; Chapelle \& Peck, 1995).

Este trabalho tem como objetivo contribuir para o conhecimento do balanço energético de Waldeckia obesa, de sua adaptação fisiológica ao ambiente e de seu papel ecológico no ecossistema marinho antártico através da avaliação do metabolismo de rotina e excreção de amônia da espécie em função da temperatura e do peso dos animais.

\section{Material e métodos}

Foram utilizados para este trabalho 169 indivíduos de Waldeckia obesa coletados na Baía do Almirantado, Illha Rei George, Antártica, nos verōes de 1990, 1991 e 1992. Os animais foram coletados com armadilhas comuns colocadas a $40 \mathrm{~m}$ de profundidade e com rastreador eletrônico colocadas entre 60 e $100 \mathrm{~m}$ de profundidade, contendo isca de carne e peixe. Foram realizados experimentos nos laboratórios com temperatura controlada da Estação Antártica Brasileira "Comandante Ferraz", Ilha Rei George e no Laboratório de Ecologia Polar do Instituto Oceanográfico da USP, em São Paulo. $\mathrm{Na}$ Antártica, os animais foram mantidos em tanques com troca constante de água por pelo menos 7 dias antes de serem empregados nos experimentos. Em São Paulo, os animais foram deixados em repouso por cerca de 3 meses, após o transporte, para perfeita adaptação às condiçōes de cativeiro e aclimatação à temperatura experimental.

Para os experimentos em São Paulo, os animais foram transportados da Antártica em sacos plásticos de 5 litros, contendo 3 litros de água do mar filtrada e insuflados com oxigênio puro. Em cada saco foram colocados cerca de 50 animais. Os sacos foram acondicionados em caixas forradas com isopor, no interior das quais foi colocado gelo para manutenção da temperatura. Essa forma de transporte vem sendo empregada desde 1989, com excelentes resultados. Em São Paulo, os animais foram mantidos em aquários de 40 litros com aeração artificial, filtro biológico e troca mensal de metade da água do mar, coletada em mar aberto na região de Ubatuba (SP), sendo alimentados com pedaços de peixe e de krill congelado. Dessa forma, espécimens de Waldeckia obesa vêm 
sobrevivendo em aquários por mais de três anos em nosso laboratório, a $0^{\circ} \mathrm{C}$, com ciclo de luz simulando o natural.

$\mathrm{O}$ consumo de oxigênio foi medido em respirômetros selados de $30 \mathrm{ml}$. Antes dos experimentos os animais foram mantidos nos respirômetros com circulação contínua de água por 6 horas, na mesma temperatura dos experimentos, para atenuar o estresse devido ao manuseio. Em seguida, o fornecimento de água foi suspenso e os respirômetros foram fechados para que os animais consumissem o oxigênio presente em volume conhecido de água, por um período que variou de 3 a 12 horas dependendo da relação do peso do animal com o volume do respirômetro. Toda água do mar utilizada foi previamente filtrada em filtro Cuno de $2 \mu \mathrm{m}$. Testes preliminares demonstraram que, nas condiçōes experimentais empregadas, as alteraçōes provocadas por organismos menores do que $2 \mu \mathrm{m}$ não são detectáveis. A diferença entre as concentraçōes de oxigênio determinadas no início e no final do confinamento representa o consumo do animal durante o período. Para minimizar o efeito da concentração do oxigênio sobre o metabolismo, a duraçâo dos experimentos foi regulada de tal modo que a concentraçāo final de oxigênio fosse sempre em torno de $75 \%$ da concentração inicial.

Para determinaçāo do oxigênio dissolvido aplicou-se o método de Winkler, modificado por Fox \& Wingficld (1938), para pequenos volumes de água. As medidas foram sempre realizadas em duplicata.

No final dos experimentos de respirometria foram retiradas amostras de água para a estimativa da excreção de amônia através do método colorimétrico de Koroleff (1970). Foram utilizadas como branco, amostras de água do mar filtrada, da mesma procedência que a utilizada para adaptar os animais e encher os respirômetros no início dos experimentos.

Na Antártica, foram realizadas 50 mediçōes de consumo de oxigênio e excreçāo de amônia à temperatura de $3^{\circ} \mathrm{C}$, com animais entre $0,04 \mathrm{~g} \mathrm{e} 0,69 \mathrm{~g}$.
Em Sāo Paulo, foram realizadas 109 mediçōes de consumo de oxigênio e de excreção de amônia, à temperatura de $\mathrm{O}^{\circ} \mathrm{C}$, com animais entre $0,12 \mathrm{~g} \mathrm{e} 0,62 \mathrm{~g}$.

Antes de serem utilizados os animais foram deixados em jejum por um período de 72 horas.

O processamento dos dados foi feito com auxílio do programa Lotus 123 para microcomputador IBM/PC adaptado para o trabalho. Foram calculados, para cada experimento, o consumo médio $\left(\mu 1 \mathrm{O}_{2} / \mathrm{h} /\right.$ indivíduo $)$ e o específico de oxigênio $\left(\mu 1 \mathrm{O}_{2} / \mathrm{mg} / \mathrm{h}\right)$ e a excreçāo média (ng.at/h/indivíduo) e específica de amônia (ng.at $/ \mathrm{mg} / \mathrm{h}$ ).

As variaçōes do consumo médio e específico de oxigênio, da excreçáo média e específica de amônia foram analisadas em funçāo da temperatura e do peso úmido dos animais.

Em todos os casos aplicou-se a análise de regressão tendo sido calculado o coeficiente de correlação $r$, utilizando-se o pacote estatístico Statgraphics (Statistical Graphics Corporation, 1985). Foi aplicada a análise de variância para cada regressāo sendo $\mathrm{F}$ a razâo entre a variância devido a regressão tendo 1 grau de liberdade e a variância residual tendo $\mathbf{n}-2$ graus de liberdade. Os valores encontrados, tanto do consumo de oxigênio quanto da excreção de amônia, foram comparados entre cada classe de peso por análises de variância e pelo teste de Newman-Keuls. O coeficiente de van't-Hoff foi utilizado para os cálculos de Q10. A relação O:N foi calculada a partir das médias de consumo de oxigênio e excreção de amônia, a $0^{\circ} \mathrm{C} \mathrm{c} \mathrm{a} 3^{\circ} \mathrm{C}$, tomadas em equivalentes atômicos.

\section{Resultados}

Nas Tabclas 1 e 2 estão os dados referentes às médias de consumo médio $(\mu \mathrm{l} / \mathrm{h})$ e específico de oxigênio $(\mu \mathrm{l} / \mathrm{mg} / \mathrm{h})$ e de excreçāo média (ng.at/h) e específica de amônia (ng.at $/ \mathrm{mg} / \mathrm{h}$ ) de Waldeckia obesa, obtidas por classe de peso dos indivíduos utilizados em experimentos

Tabela 1. Dados referentes às médias do consumo médio (ConsMed) e consumo especffico (ConsEsp) de oxigênio e da excreção média (AmMed) e especffica (AmEsp) de amônia, por classe de peso de Waldeckia obesa, na temperatura de $0^{\circ} \mathrm{C}$

\begin{tabular}{|c|c|c|c|c|c|}
\hline $\begin{array}{l}\text { Peso } \\
\text { (g) }\end{array}$ & N & $\begin{array}{l}\text { ConsMed } \\
\left(\mu \mathrm{lO}_{2} / \mathrm{h}\right)\end{array}$ & $\begin{array}{c}\text { ConsEsp } \\
\left(\mu 10_{2} / \mathrm{mg} / \mathrm{h}\right)\end{array}$ & $\begin{array}{c}\text { AmMled } \\
\text { (ng.at/h) }\end{array}$ & $\begin{array}{c}\text { AmEsp } \\
\text { (ng.at } / \mathrm{mg} / \mathrm{h} \text { ) }\end{array}$ \\
\hline 0,12 a 0,19 & 23 & $2,22+0,76$ & $0,014+0,005$ & $13,84+8,45$ & $0,085+0,049$ \\
\hline 0,20 a 0,29 & 29 & $2,48+0,96$ & $0,011+0,004$ & $15,82+7,25$ & $0,068+0,032$ \\
\hline 0,30 a 0,39 & 23 & $5,33+2,20$ & $0,016+0,007$ & $26,34+12,72$ & $0,078+0,040$ \\
\hline 0,40 a 0,47 & 12 & $5,96+2,12$ & $0,014+0,005$ & $36,99+18,88$ & $0,085+0,043$ \\
\hline 0,50 a 0,59 & 19 & $7,06+2,40$ & $0,013+0,004$ & $22,89+0,016$ & $0,042+0,029$ \\
\hline 0,60 a 0,62 & 03 & $10,81+1,37$ & $0,018+0,002$ & $55,34+19,61$ & $0,090+0,032$ \\
\hline
\end{tabular}


Tabela 2. Dados referentes às médias do consumo médio (ConsMed) e consumo especifico (ConsEsp) de oxigênio e da excreção média (AmMed) e especffica (AmEsp) de amônia, por classe de peso de Waldeckia obesa, na temperatura de $3^{\circ} \mathrm{C}$

\begin{tabular}{|c|c|c|c|c|c|}
\hline $\begin{array}{l}\text { Peso } \\
\text { (g) }\end{array}$ & N & $\begin{array}{l}\text { ConsMed } \\
\left(\mu\left(0_{2} / \mathrm{h}\right)\right.\end{array}$ & $\begin{array}{c}\text { ConsEsp } \\
\left(\mu \mathrm{lO}_{2} / \mathrm{mg} / \mathrm{h}\right)\end{array}$ & $\begin{array}{c}\text { Anthed } \\
\text { (ng.at/h) }\end{array}$ & $\begin{array}{c}\text { AmEsp } \\
\text { (ng.at/mg/h) }\end{array}$ \\
\hline 0,04 a 0,08 & 10 & $1,83+0,47$ & $0,033+0,007$ & $5,11+2,48$ & $0,085+0,026$ \\
\hline 0,14 a 0,16 & 10 & $4,89+1,57$ & $0,032+0,010$ & $13,71+6,05$ & $0,088+0,040$ \\
\hline 0,24 a 0,28 & 06 & $8,17+3,26$ & $0,030+0,011$ & $19,47+9,28$ & $0,074+0,036$ \\
\hline 0,31 a 0,315 & 04 & $9,27+3,05$ & $0,030+0,010$ & $25,71+7,19$ & $0,082+0,023$ \\
\hline 0,41 a 0,48 & 06 & $11,36+1,89$ & $0,025+0,003$ & $30,41+12,56$ & $0,068+0,027$ \\
\hline 0,51 a 0,58 & 06 & $12,70+2,45$ & $0,023+0,004$ & $34,72+11,54$ & $0,063+0,018$ \\
\hline 0,62 a 0,69 & 08 & $14,19+3,67$ & $0,022+0,006$ & $38,33+20,56$ & $0,059+0,033$ \\
\hline
\end{tabular}

experimentos realizados $\mathrm{a} 0^{\circ} \mathrm{Ce} a 3^{\circ} \mathrm{C}$, respectivamente. Os testes estatísticos indicam que as diferenças encontradas no consumo de oxigênio entre as duas temperaturas são significativas, enquanto que, as de excreção de amônia não são significativas.

O consumo médio de oxigênio aumentou em função do peso úmido dos animais de forma significativa. As equaçōes que expressam as regressões lineares do consumo médio $(\mathrm{Y})$ em função do peso úmido $(\mathrm{X})$ sāo $\log (Y)=\log (-2,14)+1,09 \log (X)$, para a temperatura de $0^{\circ} \mathrm{C}$ (Fig. 1), e, $\log (\mathrm{Y})=\log (-1,19)+0,84 \log (\mathrm{X})$ para a temperatura de $3^{\circ} \mathrm{C}$ (Fig. 2).

Análises de variância indicaram que o consumo específico não variou significativamente com o peso úmido dos animais, tanto a $0^{\circ} \mathrm{C}$ quanto a $3^{\circ} \mathrm{C}$. As equaçōes que expressam as regressões lineares do consumo específico (Y) em função do peso úmido $(X)$ são $\log (Y)=$ $\log (-2,14)+0,09 \log (X)$, para a temperatura de $0^{\circ} \mathrm{C}, \mathrm{e}$, $\log (Y)=\log (-1,19)-0,16 \log (X)$ para a temperatura de $3^{\circ} \mathrm{C}$.

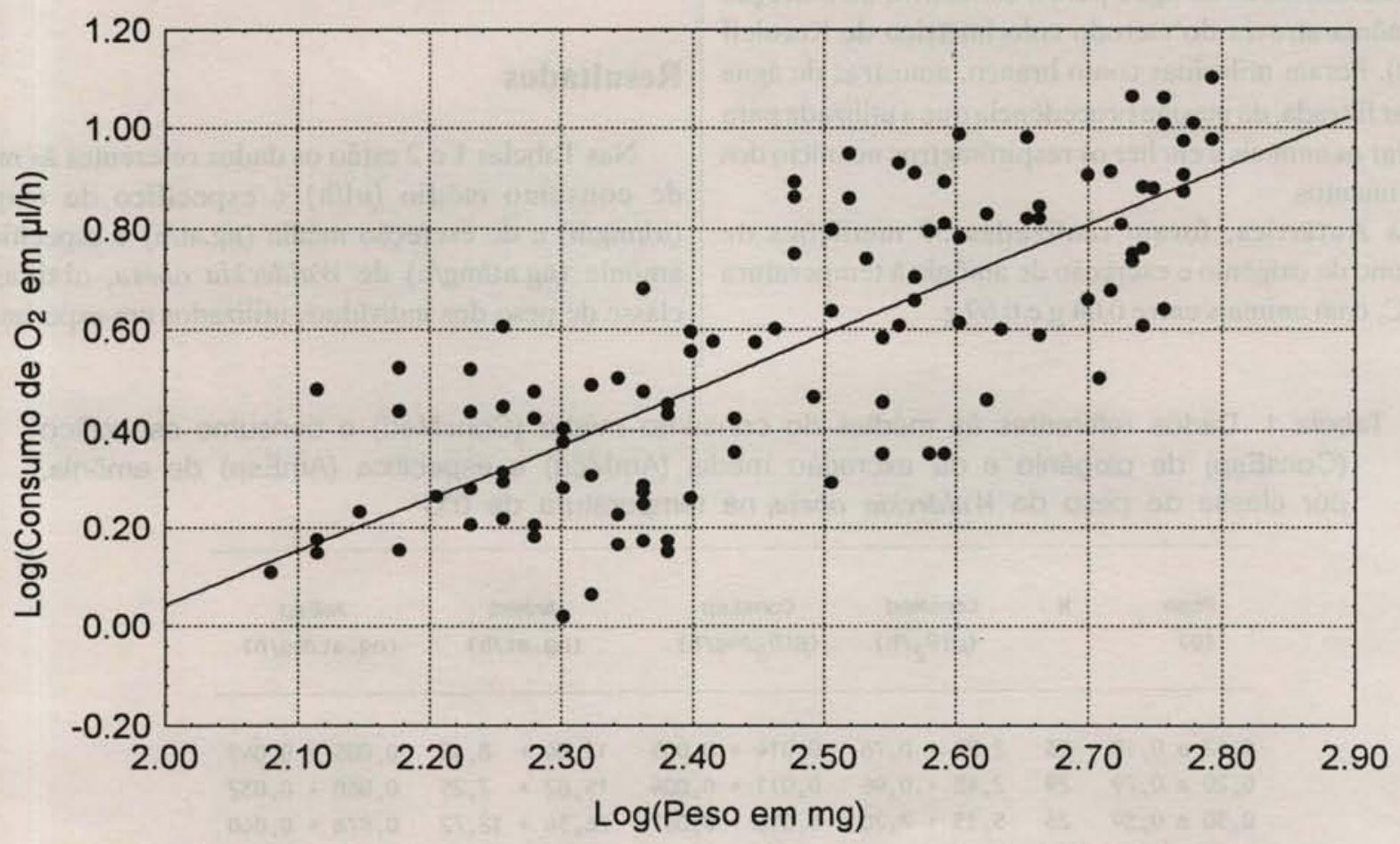

Fig. 1. Consumo médio de oxigênio (ConsMed) de Waldeckia obesa em função do peso úmido do animal, na temperatura de $0^{\circ} \mathrm{C}$. 


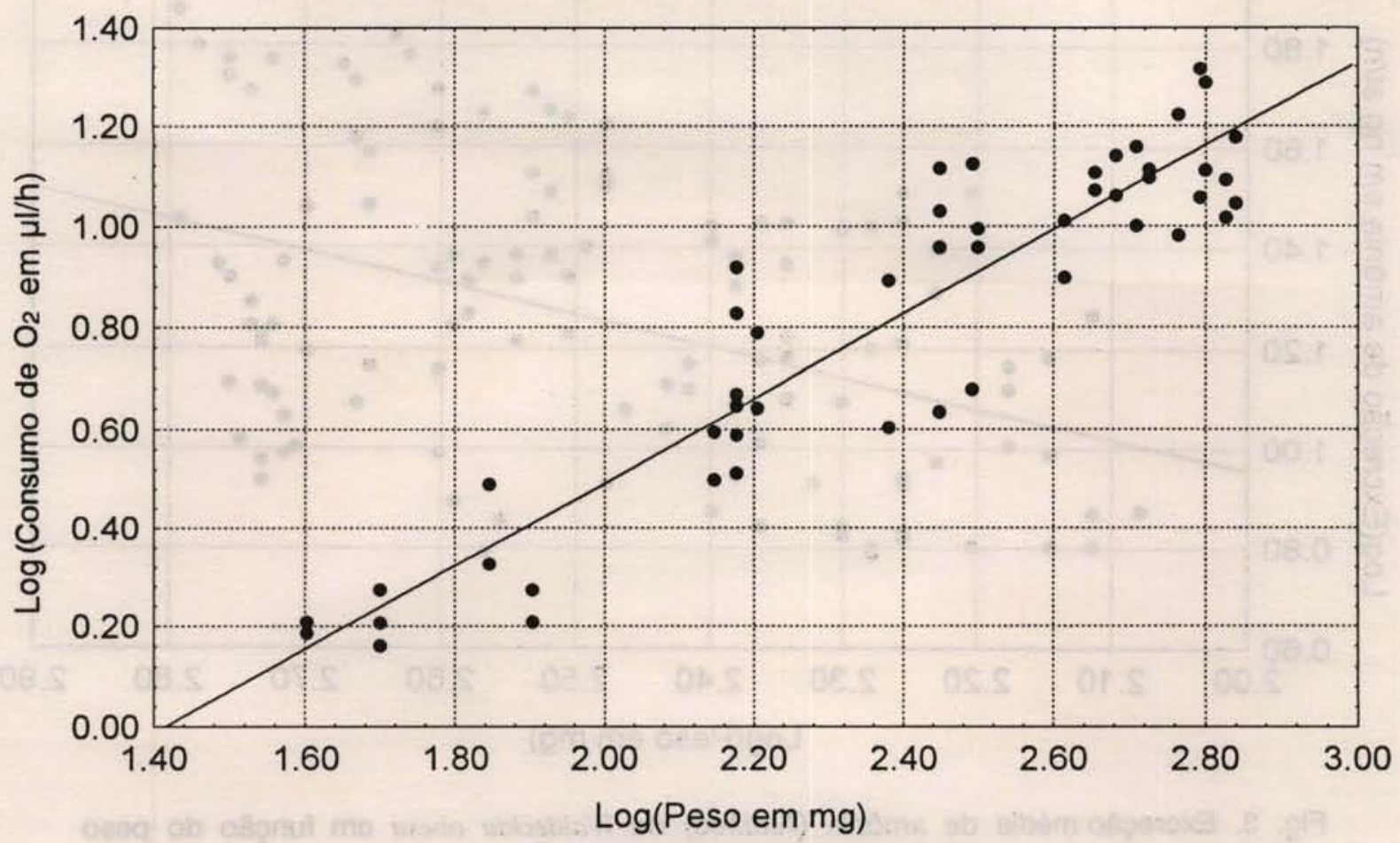

Fig. 2. Consumo médio de oxigênio (ConsMed) de Waldeckia obesa em função do peso úmido do animal, na temperatura de $3^{\circ} \mathrm{C}$.

A $0^{\circ} \mathrm{C}$, o consumo médio de oxigênio variou de 2,22 $\mu \mathrm{l} / \mathrm{h}$ para animais entre $120 \mathrm{mg}-190 \mathrm{mg}$, até $10,81 \mu \mathrm{l} / \mathrm{h}$ para animais entre $600 \mathrm{mg}-620 \mathrm{mg}$. A essa mesma temperatura, o consumo específico variou de 0,011 $\mu \mathrm{l} / \mathrm{mg} / \mathrm{h}$ para animais entre $200 \mathrm{mg}-290 \mathrm{mg}$ até 0,018 $\mu \mathrm{l} / \mathrm{mg} / \mathrm{h}$ para animais entre $600 \mathrm{mg}-620 \mathrm{mg}$.

A $3^{\circ} \mathrm{C}$, o consumo médio de oxigênio variou de 1,83 $\mu \mathrm{l} / \mathrm{h}$ para animais entre $0 \mathrm{mg}-80 \mathrm{mg}$ até $14,19 \mu \mathrm{l} / \mathrm{h}$ para animais entre $620 \mathrm{mg}-690 \mathrm{mg}$. A essa mesma temperatura, o consumo específico variou de 0,033 $\mu \mathrm{l} / \mathrm{mg} / \mathrm{h}$ para animais entre $40 \mathrm{mg}-80 \mathrm{mg}$ até 0,022 $\mu \mathrm{l} / \mathrm{mg} / \mathrm{h}$ para animais entre $620 \mathrm{mg}$ a $690 \mathrm{mg}$.

O Q10 calculado a partir das médias do consumo de oxigênio a $0^{\circ} \mathrm{C}$ e $3^{\circ} \mathrm{C}$ foi de 6,95 .

A excreçāo média de amônia aumentou significativamente em função do peso úmido dos animais. As equaçōes que expressam as regressōes lineares da excreção média de amônia (Y) em função do peso úmido $(X)$ são $\log (Y)=\log (-0,29)+0,62 \log (X)$, para a temperatura de $0^{\circ} \mathrm{C}$ (Fig. 3), e, $\log (\mathrm{Y})=\log (-0,86)+$ $0,87 \log (X)$ para a temperatura de $3^{\circ} \mathrm{C}$ (Fig. 4).

A excreção específica de amônia apresentou tendência a diminuir em função do peso úmido dos animais, tanto a $0^{\circ} \mathrm{C}$ quanto a $3^{\circ} \mathrm{C}$, se bem que, de modo geral, essa diminuição não foi estatisticamente significativa. As equações que expressam as regressões lineares da excreção específica de amônia $(\mathrm{Y}) \mathrm{em}$ função do peso úmido $(X)$ são $\log (Y)=\log (-0,29)-0,38$ $\log (X)$, para a temperatura de $0^{\circ} \mathrm{C}, \mathrm{e}, \log (\mathrm{Y})=$ $\log (-0,86)-0,13 \log (X)$ para a temperatura de $3^{\circ} \mathrm{C}$.

A $0^{\circ} \mathrm{C}$, a excreção média de amônia variou de 13,84 ng.at/h para animais entre $120 \mathrm{mg}-190 \mathrm{mg}$ até 55,34 ng.at/h para animais entre $600 \mathrm{mg}-620 \mathrm{mg}$. A essa mesma temperatura, a excreção específica de amônia variou de $0,090 \mathrm{ng} \cdot \mathrm{at} / \mathrm{mg} / \mathrm{h}$ para animais entre $600 \mathrm{mg}$ - 620 $\mathrm{mg}$ até $0,042 \mathrm{ng} \cdot \mathrm{at} / \mathrm{mg} / \mathrm{h}$ para animais entre $500 \mathrm{mg}-590$ $\mathrm{mg}$.

A $3^{\circ} \mathrm{C}$, a excreção média de amônia variou de 5,11 ng.at/h para animais entre $40 \mathrm{mg}-80 \mathrm{mg}$ até $38,33 \mathrm{ng} . \mathrm{at} / \mathrm{h}$ para animais entre $620 \mathrm{mg}-690 \mathrm{mg}$. A essa mesma temperatura, a excreçāo específica de amônia variou de $0,088 \mathrm{ng} \cdot \mathrm{at} / \mathrm{mg} / \mathrm{h}$ para animais entre $140 \mathrm{mg}-160 \mathrm{mg}$ até $0,059 \mathrm{ng} . \mathrm{at} / \mathrm{mg} / \mathrm{h}$ para animais entre $620 \mathrm{mg}-690 \mathrm{mg}$.

$\mathrm{O} \mathrm{Q}_{10}$ calculado a partir das médias da excreção de amônia a $0^{\circ} \mathrm{C}$ e $3^{\circ} \mathrm{C}$ foi de 0,83 .

Os valores de $\mathrm{O}: \mathrm{N}$, calculados a partir das médias de consumo de oxigênio e excreção de amônia, foram de 17,62 a $0^{\circ} \mathrm{C}$ e de 33,21 a $3^{\circ} \mathrm{C}$. 


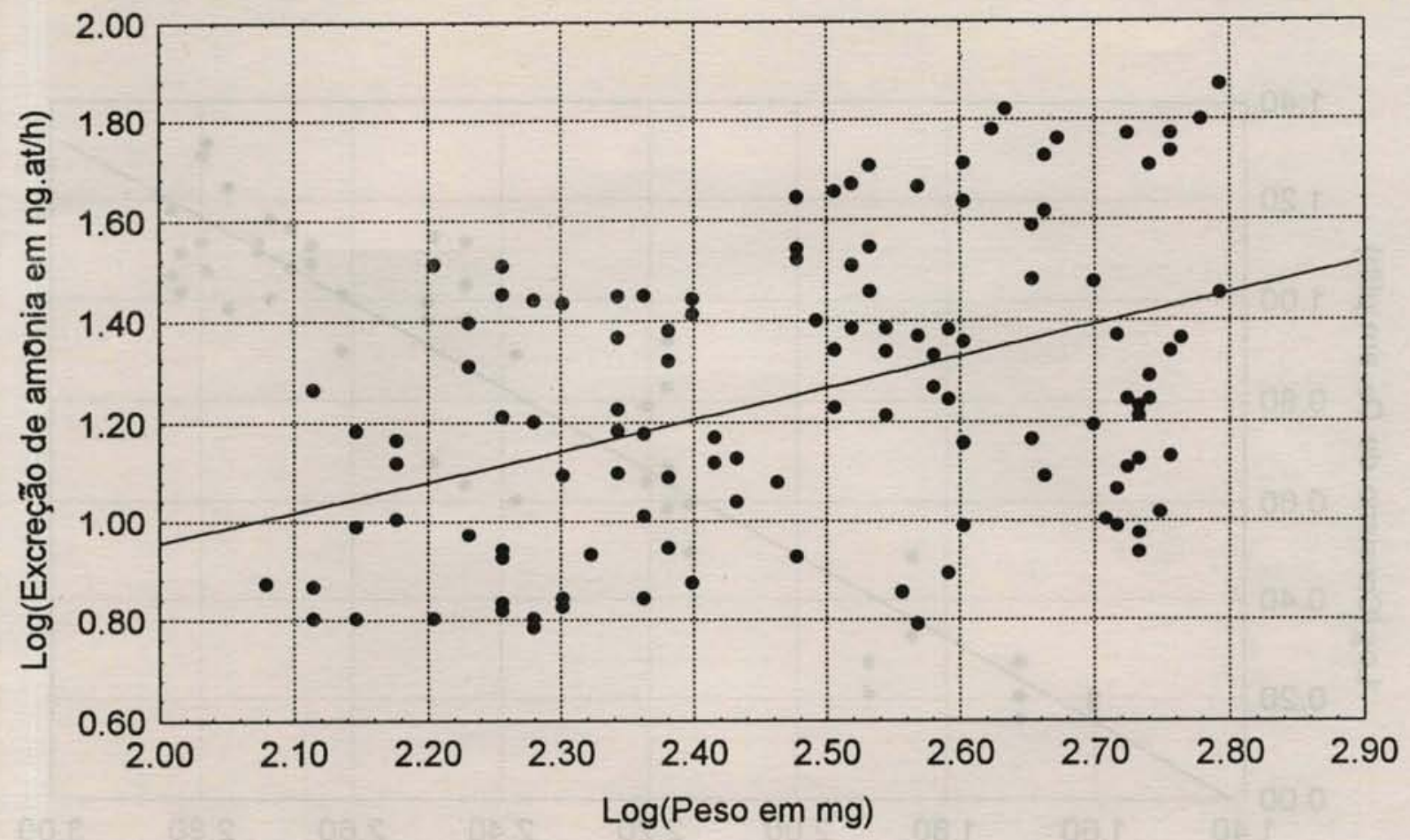

Fig. 3. Excreção média de amônia (AmMed) de Waldeckia obesa em função do peso úmido do animal, na temperatura de $0^{\circ} \mathrm{C}$.

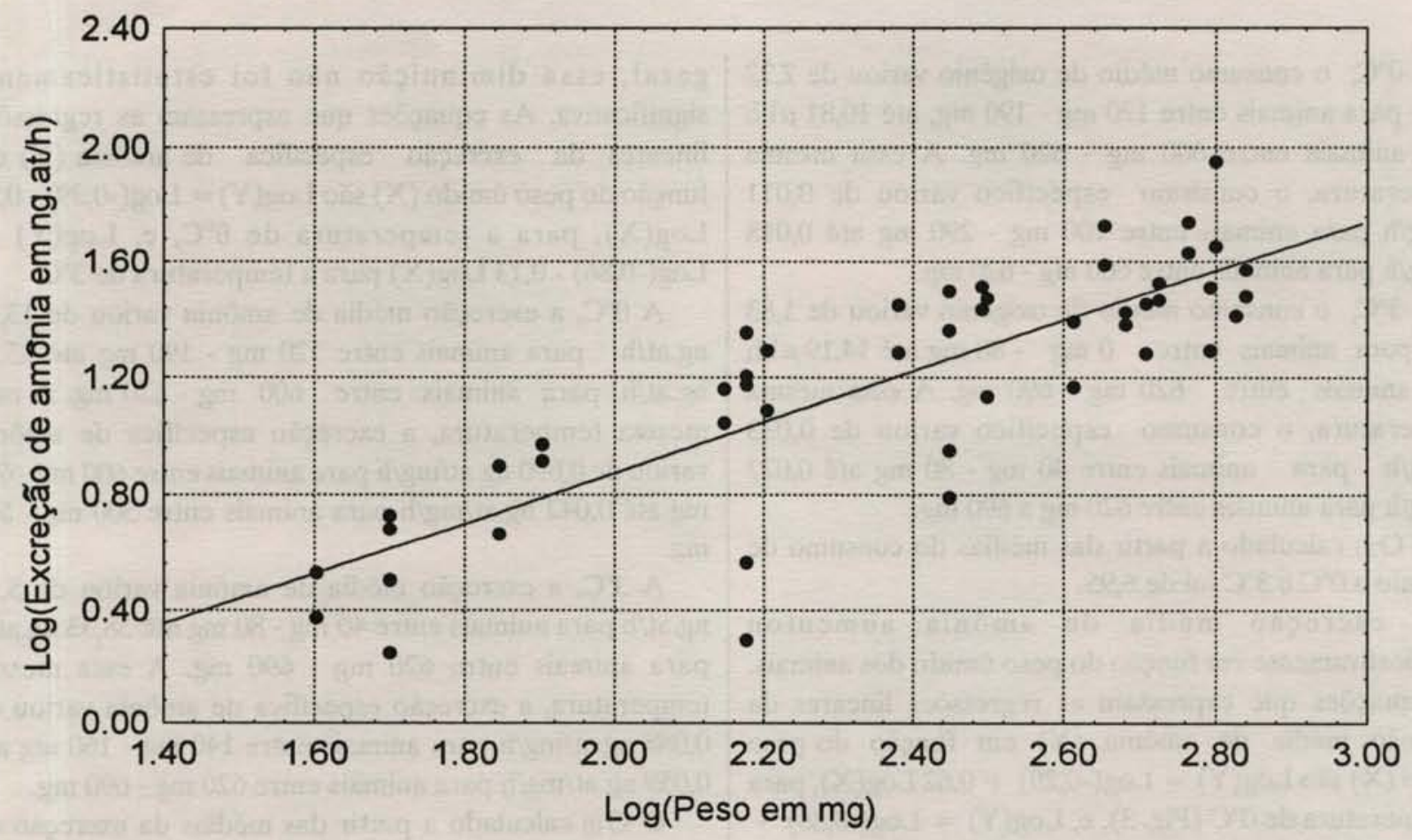

Fig. 4. Excreção média de amônia (AmMed) de Waldeckia obesa em função do peso úmido do animal, na temperatura de $3^{\circ} \mathrm{C}$. 


\section{Discussão}

O efeito da temperatura no metabolismo de animais pecilotérmicos, em geral, e de crustáceos, em particular, é bastante conhecido (Aarset \& Aunaas, 1990; Klekowski et al., 1973). Scholander et al. (1953) afirmaram que espécies de regiōes polares desenvolveram adaptaçōes metabólicas para compensar o efeito das baixas temperaturas. Segundo esses autores, animais polares apresentariam metabolismo mais elevado quando em comparação com espécies de hábitos similares de regiōes temperadas a fim de manter níveis de atividade adequados para sua sobrevivência. Essa teoria foi endossada por Wohlschlag $(1960 ; 1964)$ e corroborada por diversos outros trabalhos, incluindo com crustáceos (Armitage, 1962; Rakusa-Suszczewski, 1982). A esse fenômeno deu-se o nome de "adaptação metabólica ao frio". Opalinski (1979) chegou à conclusão de que as taxas metabólicas de anfípodas antárticos são, em média, 1,6 vezes maiores do que as de anfípodas de regiōes temperadas e 1,2 vezes maiores do que as de águas frias de outras regiōes.

Entretanto, trabalhos posteriores indicaram que as afirmaçōes feitas anteriormente nâo são confiáveis, tendo sido originadas principalmente por problemas metodológicos. Devido à grande variabilidade dos resultados encontrados, alguns autores (e.g. Rakusa-Suszczewski, 1982) sugeriram que a compensação metabólica ao frio seria um processo facultativo, ocorrendo em algumas espécies e não em outras. Entretanto, um fator limitante a ser levado em consideração é de que é muito difícil realizar comparações válidas do metabolismo entre as espécies sem que estas tenham hábitos e níveis de atividade semelhantes (Everson, 1977). Além disso, em muitos trabalhos realizados com anfípodas polares, talvez devido a escassez do tempo disponível para os experimentos durante as expediçōes $\mathrm{e}$ às dificuldades das condiçōes de laboratório, o período de aclimatação às temperaturas experimentais e o período de repouso dos animais para evitar o estresse decorrente das coletas sâo, geralmente, exíguos. No caso deste trabalho, foi empregado um período de 7 dias de aclimatação nos experimentos realizados na Antártica, e de três meses para os animais empregados no Laboratório de Ecologia Polar, em São Paulo, procurando evitar o estresse decorrente do transporte. O mesmo período de tempo foi utilizado por Chapelle \& Peck (1995) em experimentos de metabolismo de Waldeckia obesa em função do período de jejum.

Chapelle \& Peck (1995) obtiveram um valor de 2,54 $\mu \mathrm{l} / \mathrm{h}$ de consumo de oxigênio para animais de $0,295 \mathrm{~g}$ de peso úmido, em animais com 60 dias de jejum, a $0^{\circ} \mathrm{C}$. Para animais de peso correspondente, à mesma temperatura,
Rakusa-Suszczewski \& Lach (1991) obtiveram valores próximos a $8 \mu \mathrm{l} / \mathrm{h}$. Chapelle \& Peck (1995) propuseram que as diferenças encontradas se justificariam pelos cuidados tomados para evitar a influência dos processos metabólicos decorrentes da digestão e do estresse, durante seus experimentos. As taxas metab6́licas obtidas por esses autores para Waldeckia obesa foram as mais baixas já registradas para um anfípoda antártico. Nesse sentido, os autores supuseram que esses baixos valores poderiam ser devidos a diversos fatores tais como a grande quantidade de cutícula da espécie em comparação com outros anfípodas antárticos, ao longo período de jejum e às condições rigorosamente controladas dos experimentos. Os autores também afirmam que apesar das dificuldades de comparação já salientadas não existe nada que indique a existência do fenômeno chamado "adaptação metabólica ao frio" nessa espécie, e provavelmente em todas as espécies polares. Nossos dados vêm de encontro a essa afirmativa. Para animais a $0^{\circ} \mathrm{C}$, com peso entre $0,20 \mathrm{~g}-0,29 \mathrm{~g}$, o consumo médio de oxigênio obtido foi $2,48 \mu \mathrm{l} / \mathrm{h}$, praticamente o mesmo obtido por Chapelle \& Peck (1995), para a mesma classe de peso e mesma temperatura experimental. Os animais empregados nos nossos experimentos a $0^{\circ} \mathrm{C}$ também foram aclimatados por 3 meses e os cuidados para evitar o estresse foram tomados. A alimentaçāo foi suspensa 72 horas antes dos experimentos, além do período de adaptação ao respirômetro de mais 6 horas. Parece que esse período é suficiente para evitar o efeito dos processos de digestão sobre as taxas metabólicas já que os resultados foram praticamente os mesmos que os obtidos por Chapelle \& Peck (op. cit.) para animais deixados 60 dias em jejum. Waldeckia obesa é uma espécie adaptada a permanecer longos períodos em jejum sendo que animais mantiveram-se vivos, em boas condiçōes, por até 18 meses sem alimento (Coleman, 1991). Nesse sentido, essa espécie, que normalmente já possui taxas metabólicas muito baixas, deve ser adaptada para manter seu metabolismo a níveis adequados para a sobrevivência uma vez atingido seus limites mínimos.

O metabolismo de rotina de Waldeckia obesa encontrado neste trabalho e por Chapelle \& Peck (1995) é 2,4 a 18 vezes menor do que o encontrado para anfípodas antárticos por autores que consideraram seus resultados como decorrentes do fenômeno de adaptação metabólica ao frio. O recente, mas já clássico trabalho de Clarke (1991) considera que, com exceção do caso especial de evitar o congelamento, a adaptação à temperatura de animais antárticos engloba todos os aspectos da fisiologia do organismo que o possibilita viver em condições polares. Esta é uma forma de homeostase que deveria ser analisada ao nível molecular. Estudos de processos complexos e integrados, tais como crescimento e respiração, não fornecem necessariamente 
informaçōes úteis a respeito de adaptação ao frio e, nesse sentido, deveriam ser abandonados. Os autores salientam que o metabolismo de um animal é um conjunto de processos e deveria ser avaliado como uma medida de custo energético. $\mathrm{O}$ estudo das variações das taxas metabólicas de um organismo tem sua aplicação mais eficaz nas estimativas de fluxo de energia no ecossistema. Clarke (op. cit.) conclui que resultados recentes, contrariamente ao que se pensava, indicam que os anfípodas polares possuem, em geral, taxas metabólicas bem menores que as de animais similares de regiōes temperadas. Mesmo assim, o autor considera que esse fenômeno não deveria ser associado diretamente às baixas temperaturas no sentido direto da limitação termodinâmica. As baixas taxas de consumo de oxigênio parecem estar relacionadas com a demanda reduzida de ATP para o "turnover" das proteínas, com atividades da bomba iônica e outros aspectos do metabolismo basal.

O consumo de oxigênio de Waldeckia obesa aumentou acentuadamente com a temperatura, em todas as classes de peso. $O Q_{10}$ calculado a partir das médias de consumo, obtidas para as temperaturas de $0^{\circ} \mathrm{C} \mathrm{e} 3^{\circ} \mathrm{C}$, foi igual a 6,95 . Altos valores de $\mathrm{Q}_{10}$ parecem ser comuns em anfípodas antárticos tendo sido documentados para diversas espécies e sendo considerados, há muito tempo, como uma tendência geral nas respostas metabólicas de anfípodas polares (Aarset \& Aunaas, 1990). Valores tão altos quanto 71,4 foram documentados para juvenis de Paramoera walkeri por Klekowski et al. (1973). Diversos fatores podem influenciar o Q10 tais como período de aclimatação, estádio de maturidade, condiçōes fisiológicas, estresse, etc. podendo resultar em valores exageradamente elevados. Mesmo desconsiderando-se os excessos, parece que $\circ \mathrm{Q}_{10}$ é relativamente mais alto nas faixas de temperatura nas quais os crustáceos antárticos estāo adaptados quando em comparação com espécies de águas mais quentes. Talvez fosse válido supor que o conceito de $Q_{10}$, de que as taxas metabólicas normalmente duplicam com o incremento de $10^{\circ} \mathrm{C}$ na temperatura, foi desenvolvido com espécies de regiōes temperadas adaptadas a suportar uma variação bem mais ampla de temperatura do que espécies polares. Diversos anfípodas antárticos, tais como Waldeckia obesa, normalmente vivem em uma faixa muito estreita de variação de temperatura ambiental. Desse modo, variaçōes aparentemente pequenas dentro dessa faixa devem afetar o metabolismo de anfípodas polares mais intensamente do que diferenças de mesma magnitude em animais de zonas temperadas. Clarke (1988) afirma que é particularmente inapropriado aplicar o $Q_{10}$ para animais antárticos que vivem em ambientes com flutuações anuais de temperatura pequenas e que possuem uma faixa de tolerância térmica relativamente estreita. Além disso, a temperatura de $3^{\circ} \mathrm{C}$ empregada nos experimentos está próxima aos limites máximos naturais para a espécie. A esse aumento nas taxas de consumo de oxigênio segue-se, em geral, um rápido declínio quando a temperatura é elevada a níveis mais altos próximos ao limite letal das espécies (Rakusa-Suszczewski, 1975).

Por outro lado, o mesmo parece não ocorrer em relaçâo às taxas de excreção de amônia. Tal como no caso encontrado para Waldeckia obesa, resultados recentes indicam que, em geral, o consumo de oxigênio de anfípodas polares é mais sensível às variaçōes da temperatura do que a excreção de amônia (Aarset \& Aunaas, 1990). No caso de Waldeckia obesa as taxas de excreção de amônia não demonstraram variação acentuada com a elevaçâo da temperatura apresentando, inclusive, tendência a diminuir. As taxas de excreção de amônia são comumente bastante variáveis por se tratar de um processo muito complexo sujeito à influência de fatores de difícil controle, incluindo o tipo de substrato utilizado para oxidação de acordo com fatores internos e externos. Um aumento na taxa de excreção correlacionada à elevação da temperatura sugere maior eficiência de digestão e assimilação em temperaturas mais altas, ocorrendo em muitas espécies de crustáceos (Aarset \& Aunaas, op. cit.). Entretanto, em outros casos, como por exemplo no Estágio $\mathrm{V}$ do copepodito de Calanus glacialis, a excreção é independente da temperatura na faixa entre $-1,7^{\circ} \mathrm{C}$ a $1^{\circ} \mathrm{C}$ (Aarset \& Aunaas, op. cit.).

As taxas de 0:N como indicadoras da condição de invertebrados marinhos em relação ao tipo de substrato utilizado como combustível para o metabolismo vêm sendo empregadas por inúmeros autores (e.g., Conover \& Corner, 1968; Ikeda, 1977; Mayzaud \& Conover, 1988; Chapelle et al., 1994). Taxas entre 3 a 16 são consideradas como resultantes da utilização de substrato puramente proteico no catabolismo aeróbico; desse valor a 60 , como de substrato constituído de misturas de proteínas e lipídios em diferentes proporçōes, e, acima de 60 , como de substrato puramente lipídico (Mayzaud \& Conover, 1988). O tipo de substrato utilizado pelos crustáceos marinhos depende da espécie e de diversos fatores bióticos e abióticos cujos efeitos são bastante variados (Mayzaud, 1976; Ikeda, 1977; Quetin et al., 1980).

Os resultados obtidos neste trabalho indicam que Waldeckia obesa parece utilizar normalmente como substrato para o catabolismo uma mistura de proteínas e lipídeos, em diferentes proporções. A mesma tendência foi também observada por outros autores (Chapelle $e t$ al., op. cit.). $\mathrm{A} 0^{\circ} \mathrm{C}, \mathrm{o}$ valor de $\mathrm{O}: \mathrm{N}$ apresentado por Chapelle et al. (op. cit.) para animais alimentados e o deste trabalho para animais em jejum de 72 horas são bastante semelhantes $(16,90$ e 17,62 , respectivamente). Com o aumento da temperatura, possivelmente devido a uma maior demanda de energia decorrente da aceleração do 
metabolismo, a proporção de utilização de lipídios tem a tendência a aumentar $\left(33,21\right.$ a $\left.3^{\circ} \mathrm{C}\right)$. Da mesma forma, o período prolongado de 64 dias de jejum, a $0^{\circ} \mathrm{C}$, leva a um deslocamento da mistura utilizada também em direção a lipídios, provavelmente devido a depleção do substrato preferencial (43,4 in Chapelle et al., op. cit.). Ressalta-se, entretanto, que para obtenção de resultados realísticos que reflitam o que ocorre em populações naturais os animais nāo devem ser mantidos em jejum por mais de 3 dias antes das mediçōes (Lehtonen, 1994).

O consumo médio de oxigênio variou acentuadamente com o peso do animal tanto a $0^{\circ} \mathrm{C}$ quanto a $3^{\circ} \mathrm{C}(\mathrm{b}=1,09$ e 0,84 , respectivamente). Para crustáceos em geral, os valores de b normalmente variam entre $0,67 \mathrm{e}$ 1,0 sendo, em média, em torno de 0,80 (Winberg, 1956; Armitage, 1962). Entretanto, cumpre ressaltar que valores muito baixos de até 0,34 foram também encontrados (Opalinski \& Jazdzewski, 1978). Parece mais correto supor que os valores de b para anfípodas polares, da mesma forma que para a maioria dos organismos, são na realidade uma característica específica sendo bastante variáveis intra- específicamente; um único valor não deve ser considerado como representante de todo um grupo (Hoss, 1968; Elliot, 1976).

Em relação à excreção média de amônia, Chapelle et al. (1994) encontraram, para Waldeckia obesa de $280 \mathrm{mg}$ de peso úmido recém-alimentada, o valor de $41 \mathrm{ng} . a t / \mathrm{h} \mathrm{e}$, após 64 dias de jejum, de $8 \mathrm{ng}$.at/h. Para animais de peso similar, encontramos o valor médio de $15,82 \mathrm{ng}$.at/h, após 72 horas de jejum. O valor encontrado em nosso trabalho indica que a excreçāo parece diminuir, em 72 horas, para cerca de $1 / 3$ quando comparada a de animais alimentados, mas, naturalmente, está acima do encontrado para animais em jejum prolongado.

Por outro lado, o consumo específico de oxigênio (ou seja, por grama de peso) não variou significativamente com o peso dos animais. É bastante comum, mas não obrigatório, que o consumo específico diminua com o aumento do peso e do tamanho do animal, tanto do ponto de vista inter quanto intra-específico. Entretanto, no caso de Waldeckia obesa os resultados obtidos são esperados. Primeiramente, apesar de termos utilizado nos experimentos animais de diversos tamanhos, não foram empregados aqueles com peso inferior a $40 \mathrm{mg}$, por serem muito pequenos para a metodologia aplicada. Caso fossem utilizados os resultados de consumo específico poderiam ter sido diferentes. Waldeckia obesa é uma espécie que normalmente apresenta metabolismo muito baixo. Além disso, valores de b próximos a 1, referentes às variações do consumo médio em função do peso, estão dentro dos valores comumente encontrados para anfípodas, indicando não haver variaçōes significativas no consumo específico de algumas espécies. Em relação à excreção específica de amônia houve uma tendência a diminuição com o aumento do peso mas a dispersão dos valores encontrados foi muito grande, tornando-a pouco significativa.

\section{Conclusões}

Os dados obtidos demonstram a importância da metodologia na estimativa de parâmetros metabólicos, tendo em vista a influência que os diversos fatores exercem sobre os mesmos. Cuidados para evitar o estresse, para aclimatar os animais às temperaturas experimentais e evitar os efeitos dos processos de digestão nem sempre são seriamente levados em consideração. Os dados obtidos, juntamente com aqueles apresentados por Chapelle et al. (1994) e Chapelle \& Peck (1995), indicam a fragilidade do conceito de "adaptação metabб́lica ao frio", pelo menos da maneira como foi elaborado. Os resultados já existentes a respeito da espécie fornecem subsídios importantes para a estimativa do fluxo de energia da mesma. Os dados disponíveis sobre Waldeckia obesa, apresentados neste trabalho e na literatura, permitem avaliar as variaçōes do metabolismo em função de fatores ambientais e fisiológicos importantes, ou seja, temperatura, classes de tamanho e peso, alimentação, jejum e estresse, fornecendo subsídios para a modelagem ecológica. Para melhor compreensão do papel ecológico dos anfípodas antárticos é recomendável a ampliação dos trabalhos de bioenergética com espécies chaves do ecossistema.

\section{Agradecimentos}

Agradecemos ao Programa Antártico Brasileiro, PROANTAR/CNPq, à Comissão Interministerial para os Recursos do Mar, CIRM, e ao Instituto Oceanográfico da Universidade de São Paulo, pela oportunidade, apoio logístico e financeiro para realizar este trabalho. Ao CNPq pelas bolsas de pesquisa concedidas. À Estação Antártica Brasileira "Comandante Ferraz" pela hospitalidade e apoio durante os trabalhos de campo. Ao pessoal de nosso laboratório pelo auxilio nas diversas etapas do trabalho.

\section{Referências bibliográficas}

AARSET, A. V. \& AUNAAS, T. 1990. Metabolic responses of the sympagic amphipods Gammarus wilkitzkii and Onisimus glacialis to acute temperature variations. Mar. Biol., 107:433-438. 
ARMITAGE, K. B. 1962. Temperature and oxygen consumption of Orchomonella chilensis (Heller) (Amphipoda: Gammeroidea). Biol. Bull., 123(2):225-232.

ARNAUD, P. M.; JAZDZEWSKI, K.; PRESLER, P. \& SICINSKI, J. 1986. Preliminary survey of benthic invertebrates collected by Polish Antarctic Expeditions in Admiralty Bay (King George Island, South Shetlands Islands, Antarctica). Pol. Polar Res., 7(1/2):7-24.

CHAPELLE, G. \& PECK, L. S. 1995. The influence of acclimation and substratum on the metabolism of the Antarctic amphipods Waldeckia obesa (Chevreux, 1905) and Bovallia gigantea (Pfeffer 1888). Polar Biol., 15(3):225-232.

\& CLARKE, $A$

1994. Effects of feeding and starvation on the metabolic rate of the necrophagous Antarctic amphipod Waldeckia obesa (Chevreux, 1905). J. expl mar. Biol. Ecol., 183:63-76.

CLARKE, A. 1988. Seasonality in the Antarctic marine environment. Comp. Biochem. Physiol., 90(B):461-473.

1991. What is cold adaptation and how should we measure it? Am. Zool., 31:81-92.

COLEMAN, C. O. 1991. Comparative fore-gut morphology of Antarctic amphipod (Crustacea) adapted to different food sources. Hydrobiol., 223:1-9.

CONOVER, R. J. \& CORNER, E. D. S. 1968. Respiration and nitrogen excretion by some marine zooplankton in relation to their life cycles. J. mar. biol. Ass. U.K., 48: 49-75.

DE BROYER, C. \& KLAGES, M. 1990. Studies on amphipod biology. Polarforschung, 68:113-115.

ELLIOT, J. M. 1976. Energy losses in the waste products of brown trout (Salmo trutta L.). J. Anim. Ecol., 45(2):561-580.

EVERSON, I. 1977. Antarctic marine secondary production and the phenomenon of cold adaptation. Phil. Trans. R. Soc., Lond., 279:55-66.

FOX, H. M. \& WINGFIELD, C. A. 1938. A portable apparatus for the determination of oxygen dissolved in a small volume of water. J. expl Biol., 15:437-445.
HOLETON, G. F. 1974. Metabolic cold adaptation of polar fish: fact or artefact? Physiol. Zoöl., 47(3):137-152.

HOSS, D. E. 1968. Rates of respiration of estuarine fish. In: ANNUAL CONFERENCE OF THE SOUTHEASTERN ASSOCIATION OF GAME AND FISH COMMISIONERS, $21^{\text {st }}$, New Orleans, 1967. Proceedings. New Orleans, SAGFC. p.416-423.

HUREAU, J. C. 1994. The significance of fish in the marine Antarctic ecosystems. Polar Biol., 14(5):307-313.

IKEDA, T. 1977. The effect of laboratory conditions on the extrapolation of experimental measurements to the ecology of marine zooplankton. IV. Changes in respiration and excretion rates of boreal zooplankton species maintained under fed and starved conditions. Mar. Biol., 41:241-252.

JAZDZEWSKI, K. 1993. Amphipoda. In: Rakusa-Suszczewski, S., ed. The maritime antarctic coastal ecosystem of Admiralty Bay. Warsaw, Polish Academy of Sciences, p.108-116.

KLEKOWSKI, R. Z.; OPALINSKI, K. W. \& RAKUSA-SUSZCZEWSKI, S. 1973. Respiration of intarctic Amphipoda Paramoera walkeri Stebbing during the winter season. Pol. Archs Hydrobiol., 20(2):301-308.

KOROLEFF, F. 1970. Direct determination of ammonia in natural waters as indophenol blue. Cons. Int. Explor. Mer., Information on techniques and methods for sea water analysis (and laboratory report), (3):19-22.

LEHTONEN, K. K. 1994. Metabolic effects of short-term starvation on the benthic amphipod Pontoporeia affinis Lindström from the northern Baltic Sea. J. expl mar. Biol. Ecol., 176:269-283.

MAYZAUD, P. 1976. Respiration and nitrogen excretion of zooplankton. IV. The influence of starvation on the metabolism and the biochemical composition of some species. Mar. Biol., 37:47-58.

\& CONOVER, R. J. 1988. O:N atomic ratio as a tool to describe zooplankton metabolism. Mar. Ecol.-Prog. Ser., 45: 289- 302. 
OPALINSKI, K. W. 1979. Metabolic cold adaptation in antarctic amphipods. Ekol. pol., 27(2):323-331.

\& JAZDZEWSKI, K. 1978. Respiration of some antarctic amphipods. Pol. Archs Hydrobiol., 25(3):643-655.

QUETIN, L. B.; ROSS, R. M. \& UCHIO, K. 1980. Metabolic characteristics of midwater zooplankton: ammonia excretion, O:N ratio and the effects of starvation. Mar. Biol., 59:201-209.

RAKUSA-SUSZCZEWSKI, S. 1975. Respiration and osmoregulation as the expression of the invertebrates and fishes to life under hypostenothermic conditions. Pol. Archs Hydrobiol., 22(4):521-552.

1982. The biology

and metabolism of Orchomene plebs (Hurley, 1965) (Amphipoda: Gammaridea) from McMurdo Sound Ross Sea, Antarctica. Polar Biol., 1(1):47-54.

\& KLEKOWSKI,

R. Z. 1973. Biology and respiration of the Antarctic Amphipoda (Paramoera walkeri Stebbing) in the summer. Pol. Archs Hydrobiol., 20(3):475-488.
RAKUSA-SUSZCZEWSKI, S. \& LACH, A. 1991. Respiration of Orchomene plebs (Hurley, 1965) and Waldeckia obesa (Chevreux, 1905) from Admiralty Bay (South Shetlands Islands, Antarctica). Hydrobiol., 223:177-180.

SCHOLANDER, P. F.; FLAGG, W.; WALTERS, V. \& IRVING, L. 1953. Climatic adaptation in arctic and tropical poikilotherms. Physiol. Zoöl., 26:67-69.

WINBERG, G. G. 1956. Rate of metabolism and food requirements of fishes. Fish. Res. Bd Can. Transl. ser., (194):1-253.

WOHLSCHLAG, D. E. 1960. Metabolism of an Antarctic fish and the phenomenon of cold adaptation. Ecol., 41(2):287-292.

1964. Respiratory metabolism and ecological characteristics of some fishes in McMurdo Sound, Antarctica. Antarct. Res. Ser., 1:33-62.

(Manuscrito recebido 28 agosto 1995; revisto 26 outubro 1995; aceito 29 novembro 1995) 